\title{
SPATA7 is differentially expressed in lymph node metastasis in human breast cancer.
}

Shahan Mamoor, MS ${ }^{1}$

1'shahanmamoor@gmail.com

East Islip, NY USA

Metastasis to the brain is a clinical problem in patients with breast cancer ${ }^{1-3}$. Between the breast and the brain reside the secondary lymphoid organ, the lymph nodes. We mined published microarray data ${ }^{4,5}$ to compare primary and metastatic tumor transcriptomes for the discovery of genes associated with metastasis to the lymph nodes in humans with metastatic breast cancer. We found that spermatogenesis associated 7, SPATA7, was among the genes whose expression was most different in the lymph node metastases of patients with metastatic breast cancer as compared to primary tumors of the breast. Analysis of a separate microarray dataset revealed that SPATA7 was also differentially expressed in brain metastatic tissues. SPATA7 mRNA was present at decreased quantities in lymph node metastases as compared to primary tumors of the breast. Importantly, expression of SPATA7 in primary tumors of the breast was correlated with patient distant metastasis-free survival, in lymph node positive patients but not in lymph node negative patients. Modulation of SPATA7 expression may be relevant to the biology by which tumor cells metastasize from the breast to the lymph nodes and the brain in humans with metastatic breast cancer.

Keywords: breast cancer, metastasis, brain metastasis, central nervous system metastasis, lymph node metastasis, spermatogenesis associated 7, SPATA7, systems biology of breast cancer, targeted therapeutics in breast cancer. 
One report described a 34\% incidence of central nervous system metastases in patients treated with trastuzumab for breast cancer ${ }^{2}$. More recently, the NEfERT-T clinical trial ${ }^{6}$ which compared administration of either neratinib or trastuzumab in conjunction with paclitaxel demonstrated that in a randomized, controlled setting, in breast cancer patients treated with neratinib, not only was the incidence of central nervous system recurrence significantly lower, the time to central nervous system metastasis was significantly delayed as compared to patients administered trastuzumab ${ }^{6}$. The alarmingly high rate of central nervous system metastasis described, as well as data, both anecdotal ${ }^{2}$ and from a randomized, controlled setting ${ }^{6}$ illustrating that treatment with trastuzumab may be associated with these events demands an enhanced understanding of the transcriptional makeup of brain and lymph node metastatic tissues to support identification of therapeutic targets, whether they are treatment related or not. The lymph nodes reside between the breast and the brain. We performed a global comparative analysis of lymph node metastatic tumor tissues in patients with metastatic breast cancer, compared to primary tumors and normal breast tissues ${ }^{4,5}$. We discovered transcriptome-wide differential expression of the gene encoding spermatogenesis associated 7, SPATA7, in lymph node metastatic tissues of patients with metastatic breast cancer.

\section{Methods}

We used datasets GSE10893 ${ }^{4}$ and GSE52604 $4^{5}$ for this global differential gene expression analysis of brain metastatic breast cancer in conjunction with GEO2R. GSE10893 was generated using Agilent-011521 Human 1A Microarray G4110A technology with $n=71$ primary breast tumors and $n=7$ lymph node metastases from patients with breast cancer; analysis was performed using platform GPL887. GSE52604 was generated using Agilent-014850 Whole Human Genome Microarray 4x44K G4112F with $n=10$ normal breast tissues and $n=35$ brain metastases from patients with breast cancer; analysis was performed using platform GPL6480. The Benjamini and Hochberg method of $p$-value adjustment was used for ranking of differential expression but raw $p$-values were used to assess statistical significance of global differential expression. Log-transformation of data was auto-detected, and the NCBI generated category of platform annotation was used. A statistical test was performed to evaluate whether SPATA7 gene expression was significantly different between primary tumors of the breast and lymph node metastases in humans with breast cancer using a two-tailed t-test. For Kaplan-Meier survival analysis, we used the Kaplan-Meier plotter online tool ${ }^{7}$ for correlation of SPATA7 mRNA expression levels with distant metastasis-free survival in $n=889$ lymph node positive patients and $n=1309$ lymph node negative breast cancer patients.

\section{$\underline{\text { Results }}$} cancer.

We performed global comparative transcriptome analysis of metastatic tumor tissues of patients with metastatic breast cancer using published microarray data ${ }^{4,5}$ to describe the transcriptional landscape of metastasis in human breast cancer in an unbiased fashion and for the discovery of novel therapeutic targets.

\section{SPATA7 is differentially expressed in the lymph node metastases of patients with metastatic breast} cancer.

Through blind, systems-level analysis of published microarray data ${ }^{4}$, we identified spermatogenesis associated 7, encoded by SPATA7, as a differentially expressed gene in the lymph node metastatic tissues of humans with breast cancer (Table 1). When sorting each of the genes expressed in lymph node metastases based on significance of difference as compared to primary tumors of the breast in patients with breast cancer, SPATA7 ranked 102 out of 18034 total transcripts (Chart 1), equating to 99.4\% differential expression. Differential expression of SPATA7 in the lymph node metastases of patients with metastatic breast cancer was statistically significant (Chart $1 ; p=7.54 \mathrm{e}-04$ ). 
Differential gene expression analyses that utilize primary and metastatic tumor tissues to discover genes associated with metastasis can be challenging due to the relatively heterogenous cellular composition and constitution of the tumor as compared to normal, untransformed (benign) tissues. Thus, to attempt to validate differential transcriptome-wide differential expression of SPATA7 in metastatic tissues in human breast cancer, we queried a second microarray dataset ${ }^{5}$, here comparing normal breast tissues rather than primary tumors of the breast, to brain metastases. Again, we identified SPATA7 as a differentially expressed gene in the metastatic tissues of humans with breast cancer, here, in the brain metastatic tissues of patients with breast cancer (Chart 2). When sorting each of the genes expressed in brain metastases based on significance of difference as compared to normal breast tissues, SPATA7 ranked 12104 out of 41093 total transcripts (Chart 2), equating to $70.5 \%$ differential expression. Differential expression of SPATA7 in the brain metastases of patients with metastatic breast cancer was statistically significant (Chart 2; $p=1.16 \mathrm{e}-02$ ). Thus, differential expression of SPATA7, transcriptome-wide, in the metastatic tissues of women with metastatic breast cancer was conserved across two independent microarray datasets, both in lymph node and brain metastases. This suggests that SPATA7 differential expression in human metastatic breast cancer is a bona fide biological process and that differential expression of SPATA7 in metastatic breast cancer is shared between the brain and the lymph nodes.

SPATA7 is expressed at lower levels in the lymph node metastases of patients with metastatic breast cancer.

We obtained exact mRNA expression levels for SPATA7, in primary tumors of the breast and in lymph node metastases of patients with metastatic breast cancer to determine direction and statistical significance of change in SPATA7 expression in lymph node metastatic tissues. We observed significantly decreased expression of SPATA7 in the lymph node metastases of patients with breast cancer as compared to primary tumors of the breast: SPATA7 was expressed at $0.04 \pm 0.47$ arbitrary units (AU) in primary tumors of the breast, while it was expressed at $-0.60 \pm 0.40 \mathrm{AU}$ in lymph node metastatic tissues (Figure 1). The difference in SPATA7 mRNA levels between primary tumors of the breast and lymph node metastases was statistically significant (Figure $1 ; p=0.000795$ ).

\section{SPATA7 expression is significantly correlated with survival outcomes in human breast cancer.}

We performed Kaplan-Meier survival analysis ${ }^{7}$ in $n=889$ lymph node positive breast cancer patients and $n=1309$ lymph node negative breast cancer patients, to evaluate whether SPATA7 tumor expression was correlated with survival outcomes in breast cancer and to evaluate whether lymph node status influenced correlation of SPATA7 tumor expression with survival outcomes in human breast cancer. We observed a correlation between primary tumor expression of SPATA7 and distant metastasis-free survival (DMFS) in lymph node positive breast cancer (Figure 2). Lymph node positive patients whose primary tumors expressed low levels of SPATA7 possessed median DMFS of 30.62 months as compared to a median DMFS of 62.88 months in lymph node positive patients whose tumors expressed high levels of SPATA7. This difference in DMFS based on SPATA7 tumor expression in lymph node positive patients with breast cancer was statistically significant (Figure 2, Chart 3; logrank $p$-value: 0.0035; hazard ratio: 0.69 (0.53-0.89)). SPATA7 primary tumor expression was not correlated with distant metastasis-free survival outcomes in lymph node negative patients (Figure 2, Chart 3; logrank $p$-value: 0.24; hazard ratio: $0.86(0.67-1.1))$.

Thus, by mining published microarray data ${ }^{4,5}$ in an unbiased and systematic fashion, we identified SPATA7 as among the genes whose expression was most different, transcriptome-wide, in the lymph node metastases of patients with breast cancer, when compared to primary tumors; we observed significantly decreased expression of SPATA7 in lymph node metastases as compared to primary tumors of the breast. Further, we observed correlation between SPATA7 expression and distant metastasis-free survival in 
lymph node positive patients but not in lymph node negative patients.

\section{Discussion}

We provided evidence here that spermatogenesis associated 7, SPATA7, is among the genes whose expression is most different in the lymph node metastases of patients with metastatic breast cancer as compared to primary tumors of the breast, that SPATA7 is also differentially expressed in brain metastatic tissues in human breast cancer, that SPATA7 mRNA is present at significantly decreased quantities in lymph node metastatic tissues as compared to primary tumors of the breast, and that primary tumor SPATA7 expression is correlated with patient survival outcomes in human breast cancer in lymph node positive patients but not in lymph node negative patients. Evaluation of the effects of genetic depletion of SPATA7 in mouse models of metastatic breast cancer on metastasis to the lymph nodes and to the central nervous system is merited. Modulation of SPATA7 expression may be relevant to the processes by which breast cancer cells exit the breast, enter the vasculature and/or lymphatics, reside in the lymph nodes, evade immune clearance, breach the blood-brain barrier and colonize the brain. 


\section{$\underline{\text { References }}$}

1. Lin, N.U., Amiri-Kordestani, L., Palmieri, D., Liewehr, D.J. and Steeg, P.S., 2013. CNS metastases in breast cancer: old challenge, new frontiers.

2. Bendell, J.C., Domchek, S.M., Burstein, H.J., Harris, L., Younger, J., Kuter, I., Bunnell, C., Rue, M., Gelman, R. and Winer, E., 2003. Central nervous system metastases in women who receive trastuzumab-based therapy for metastatic breast carcinoma. Cancer, 97(12), pp.2972-2977.

3. Tsukada, Y., Fouad, A., Pickren, J.W. and Lane, W.W., 1983. Central nervous system metastasis from breast carcinoma autopsy study. Cancer, 52(12), pp.2349-2354.

4. Weigman, V.J., Chao, H.H., Shabalin, A.A., He, X., Parker, J.S., Nordgard, S.H., Grushko, T., Huo, D., Nwachukwu, C., Nobel, A. and Kristensen, V.N., 2012. Basal-like Breast cancer DNA copy number losses identify genes involved in genomic instability, response to therapy, and patient survival. Breast cancer research and treatment, 133(3), pp.865-880.

5. Salhia, B., Kiefer, J., Ross, J.T., Metapally, R., Martinez, R.A., Johnson, K.N., DiPerna, D.M., Paquette, K.M., Jung, S., Nasser, S. and Wallstrom, G., 2014. Integrated genomic and epigenomic analysis of breast cancer brain metastasis. Plos one, $9(1)$, p.e85448.

6. Awada, A., Colomer, R., Inoue, K., Bondarenko, I., Badwe, R.A., Demetriou, G., Lee, S.C., Mehta, A.O., Kim, S.B., Bachelot, T. and Goswami, C., 2016. Neratinib plus paclitaxel vs trastuzumab plus paclitaxel in previously untreated metastatic ERBB2-positive breast cancer: the NEfERT-T randomized clinical trial. JAMA oncology, 2(12), pp.1557-1564.

7. Györffy, B., Lanczky, A., Eklund, A.C., Denkert, C., Budczies, J., Li, Q. and Szallasi, Z., 2010. An online survival analysis tool to rapidly assess the effect of 22,277 genes on breast cancer prognosis using microarray data of 1,809 patients. Breast cancer research and treatment, 123(3), pp.725-731. 
Rank: 102

Probe ID: 13430

p-value: $7.54 \mathrm{e}-04$

t: 3.5057958

B: -0.5716

Gene: SPATA7

Gene name: spermatogenesis associated 7

Chart 1: SPATA7 is differentially expressed in lymph node metastatic breast cancer when comparing lymph node metastases to primary tumors of the breast.

The rank of global differential expression, the probe/transcript ID, the $p$-value with respect to differential expression transcriptome-wide, $\mathrm{t}$, a moderated t-statistic, $\mathrm{B}$, the log-odds of differential expression between the groups compared, the gene and gene name are listed in this chart.

\section{Rank: 12104}

Probe ID: A_32_P34387

p-value: $1.16 \mathrm{e}-02$

t: 2.6278115

B: -3.485556

Gene: SPATA7

Gene name: spermatogenesis associated 7

Chart 2: SPATA7 is differentially expressed in brain metastatic breast cancer when comparing brain metastases to normal breast tissues.

The rank of global differential expression, the probe/transcript ID, the $p$-value with respect to differential expression transcriptome-wide, $\mathrm{t}$, a moderated t-statistic, $\mathrm{B}$, the log-odds of differential expression between the groups compared, the gene and gene name are listed in this chart. 


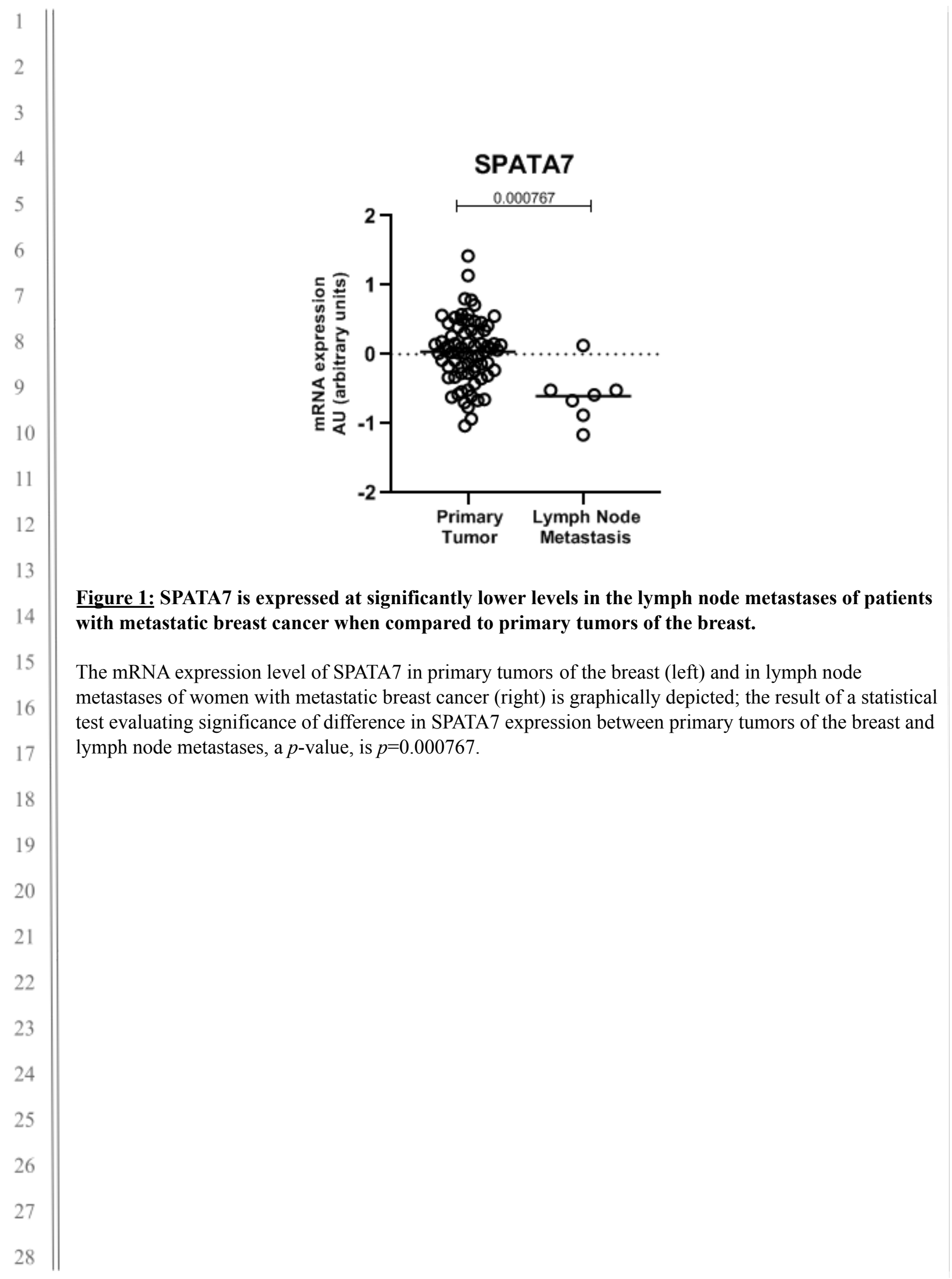




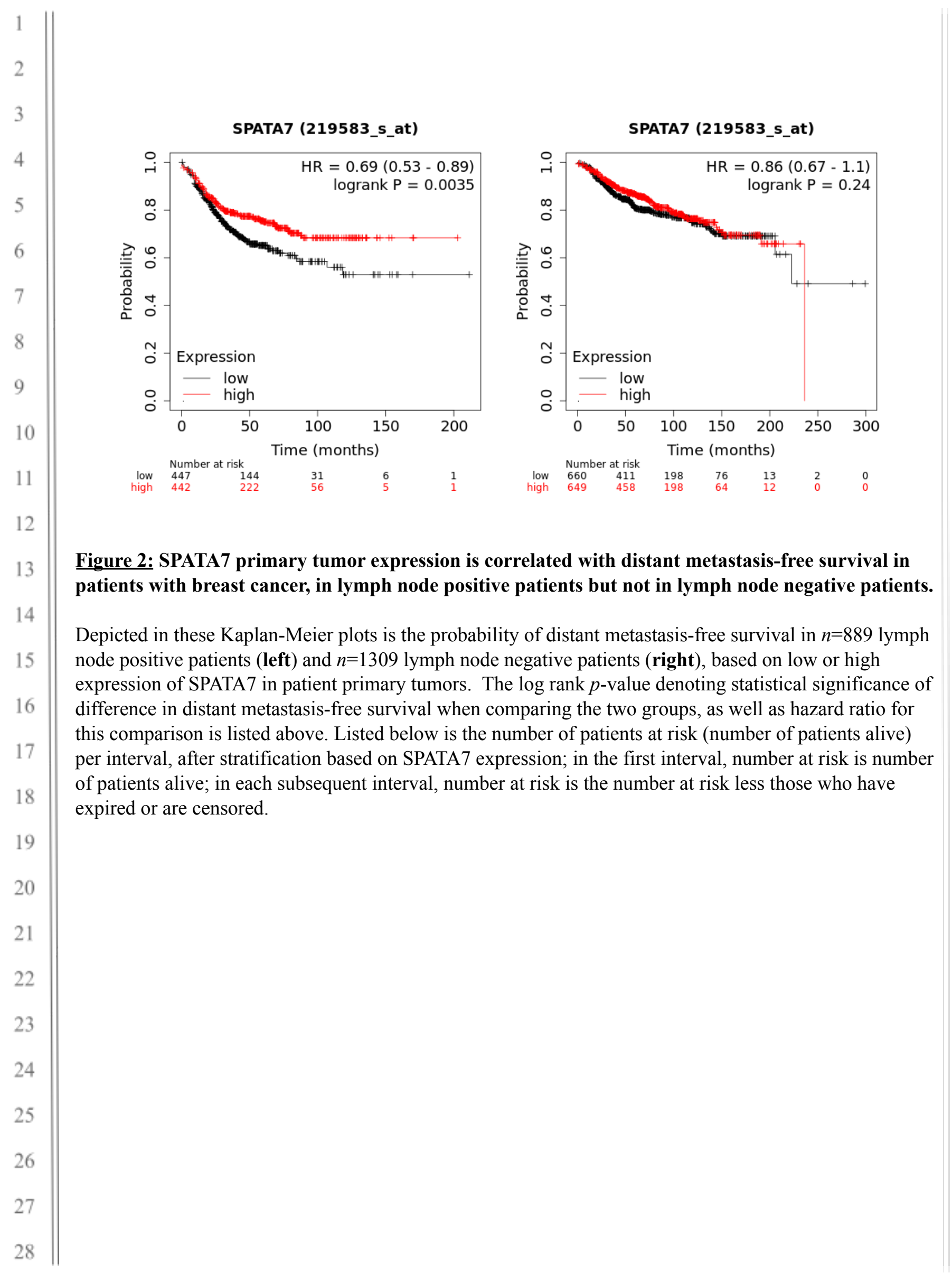




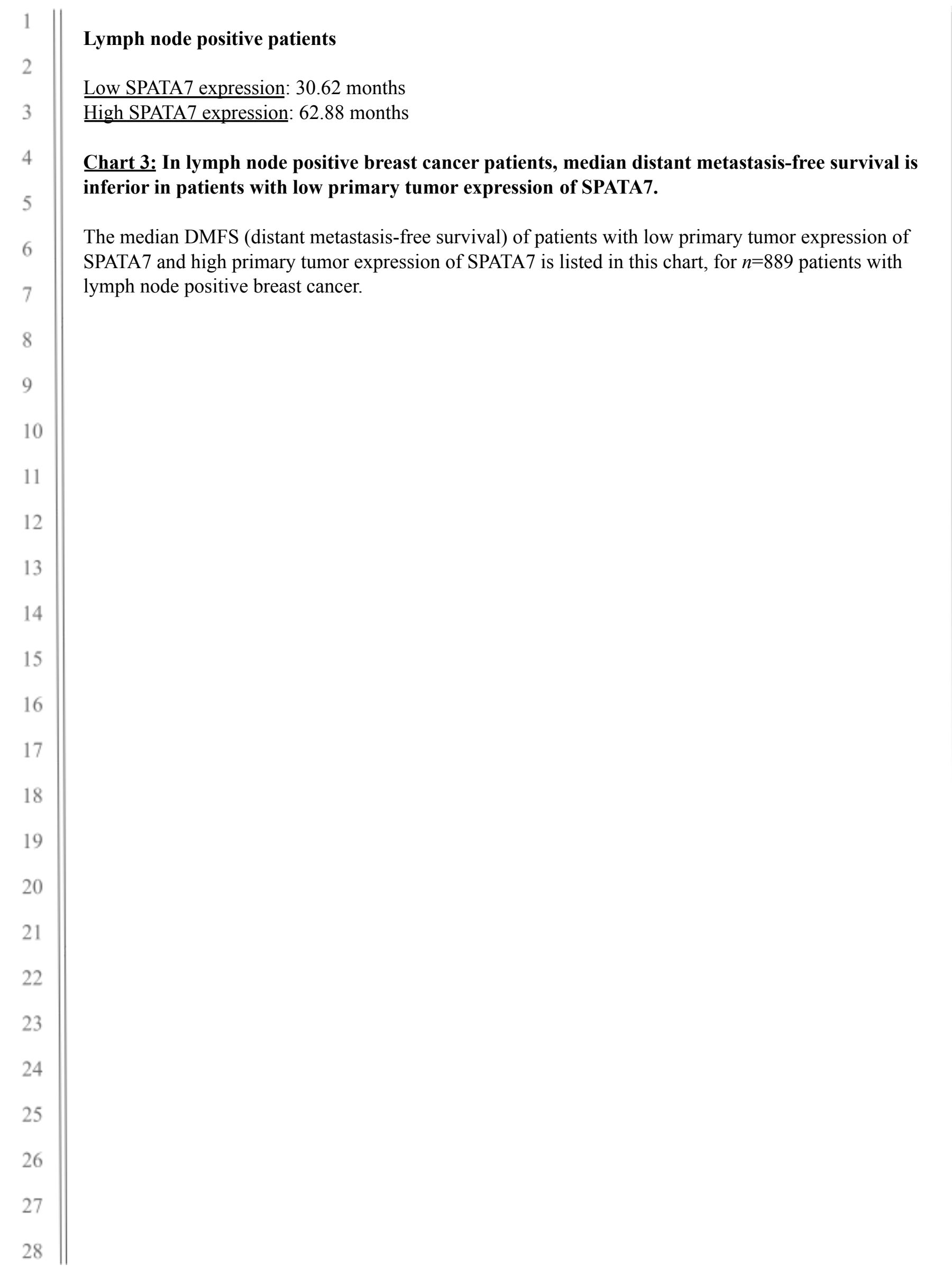

